\title{
Cepheids in Galactic Open Clusters: An All-sky Census
}

\section{Richard I. Anderson, Laurent Eyer and Nami Mowlavi}

Observatoire de Genève, CH-1290 Versoix, Swizterland email: richard.anderson@unige.ch

\begin{abstract}
We perform an all-sky search for classical (type I) Cepheids that are members of Galactic Open Clusters. Our approach is multi-dimensional, using all available spatial and kinematic parameters. The quantification of errors is crucial for this analysis, so care is taken to find adequate and realistic representations of parameter uncertainties. The data employed in the calculation are taken from published catalogues and the literature, supplemented by specific radial-velocity observations. Our work in progress is outlined here, and issues related to the inhomogeneity of cluster radii in the literature are discussed in some detail.
\end{abstract}

Keywords. (stars: variables:) Cepheids, (Galaxy:) open clusters and associations: general, stars: evolution, stars: distances, astrometry, stars: kinematics, catalogues, astronomical data bases: miscellaneous, (cosmology:) distance scale

\section{Introduction}

Cluster Cepheids (CCs) have been the object of attention for a long time, owing in part to the ability to calibrate the zero-point of the well-established period-luminosity relationship (PLR); see, e.g., Sandage \& Tammann (2006), Turner (2010). A solid determination of cluster membership for PLR calibrators is obviously of the utmost importance. Investigations of $\mathrm{CC}$ membership therefore usually take into account multiple membership constraints including position, distance and radial velocity or proper motion, as well as considerations of evolutionary status (age). Nevertheless, the membership of some CCs is disputed, for example SZ Tau in (or not) NGC 1647 (Baumgardt et al. 2000). Furthermore, studies of individual Cepheid-Cluster pairs suffer from data inhomogeneity, resulting in a lack of inter-comparability. In this paper we outline our work in progress that aims to enlarge the number of known Cluster Cepheids significantly; it is based on a self-consistent analysis of the literature and newly-observed data. We aim to avoid a direct dependence on stellar models by not considering evolutionary status as a membership constraint.

\section{Overview}

\subsection{Compilation of Literature Data}

We consider Open Clusters mentioned in Kharchenko et al. (2005) [hereafter K05] and Bukowiecki et al. (2011) [hereafter B11]. Those two catalogues were chosen because of their comparable definitions of tidal radii (see Section 3) in order to ensure comparability of our results. The compilation of cluster data and parameters by Dias et al. (2002) [from hereon DAML] was of great help for supplementing average proper motions or radial velocities. A list of classical Cepheids was compiled using the GCVS (Samus et al. 2011) and VSX (Watson 2006) catalogues. Additional information was added from many other 
sources through cross-matching, for example from the Fernie et al. (1995) database of Cepheids.

Selected high-precision radial-velocity observations were conducted at the Euler (La Silla, Chile) and the Mercator (La Palma, Canary Islands) telescopes to supplement this otherwise purely literature-based study.

\subsection{Computation of Membership Probabilities}

After making a cone search to identify CC candidates, we calculated "membership probabilities", that is, the probabilities of the null hypotheses (membership) being true for the candidates. The main assumptions made are Gaussian uncertainties, independent measurements, spherical symmetry for the clusters, accurate representation of parameters. In other words, starting from a proximity argument to investigate cluster membership, we filter out cases that are inconsistent with membership. Good cases remain, and will have to be checked individually.

The computation compares position (on-sky separation weighted by cluster radius), distance, proper motions in each direction, and median radial velocities. If $x$ is the vector of differences between Cluster and Cepheid parameters, and $\Sigma$ the sum of the covariance matrices, then we can calculate the unitless parameter $c$ according to Robichon et al. (1999) and Baumgardt et al. (2000):

$$
c=x^{\mathrm{T}} \cdot \Sigma^{-1} \cdot x
$$

where $c \sim \chi_{N}^{2}$ and $N$ denotes the degrees of freedom. We can then calculate the p-value associated with $c$ and thus obtain our "membership probability".

\section{Open Cluster Radii}

In this project, we combine very inhomogeneous data from multiple sources. That leads to various problems that cannot all be covered here in full; here we discuss solely some issues relating to cluster radii, and refer to our article (currently in preparation) for an in-depth discussion concerning all parameters.

The most extensive catalogue of Open Cluster parameters is probably the [DAML] catalogue. It is in part based on WEBDA (available at www.univie.ac.at/webda/), is repeatedly updated, and is subject to quality controls. It lists diameters for $99 \%$ of all clusters.

However, multiple definitions of "cluster diameters" exist in the literature, such as "apparent diameters" (from visual inspection), "half-light radii", "core radii", "tidal radii" and "limiting radii" (based on star counts or density profiles). Some of these may be similarly defined, yet no single and precise definition of the quantity "cluster radius" exists. As a consequence, the compilation of diameters in [DAML] cannot adhere to a single definition. That creates a problem for our analysis, since we depend on well-defined cluster radii in two ways: 1) the initial selection of Cepheids in nearby clusters is obtained through a cone search around the cluster position, within an area defined by twice the limiting cluster radius, and 2) the membership probability calculated in Eq. 2.1 depends on the radius, since we use the radius in an ad-hoc definition of a positional membership probability distribution centred around the cluster's coordinates. We thus choose to limit our list of clusters to the combined list of [K05] and [B11], whose cluster radii are similarly, but not equally, defined ("coronal" vs. "limiting" radii). Unfortunately, that results in the exclusion of around 840 clusters listed in [DAML] in favour of ensuring comparability of our results. 

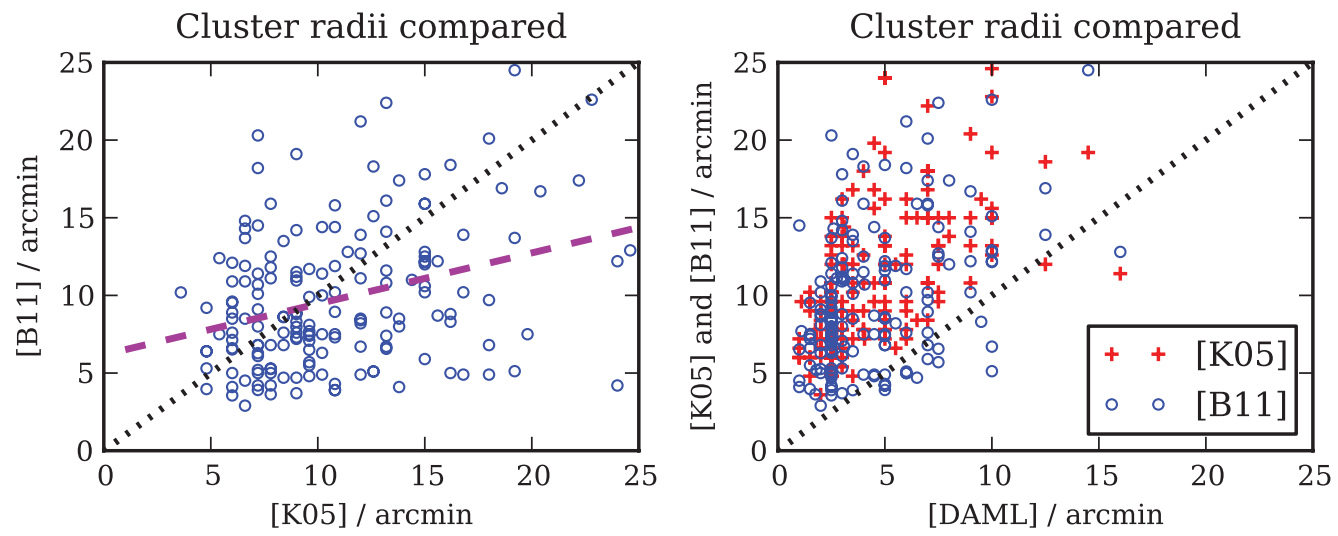

Figure 1. Left panel: Cluster radii in [B11] against those in [K05]. Dashed line indicates least squares fit for clarity. [K05] radii are on average larger with large scatter. Right panel: Comparison of [K05] and [B11] radii with apparent radii found in [DAML].

Figure 1 (left) compares the radii of 165 clusters common to [K05] and [B11], setting cluster radius $<25^{\prime}$ to avoid border issues arising from the $34^{\prime}$-limited FOV in [B11], while Figure 1 (right) compares radii in the two catalogues with apparent radii from [DAML]. Two important features should be noted: a) there is a large scatter in radii, exceeding a factor of two for a number of objects; b) [B11] radii, though based on 2MASS infrared photometry, are on average smaller than the optically-determined [K05] radii, as can be seen from the least-squares fit indicated by the dashed line. That is very interesting, as it shows the opposite trend to what was noted in [B11]. We suggest the following explanation: Both the fainter magnitude limit of 2MASS photometry and its enhanced sensitivity to red low-mass stars compared to optical photometry used by [K05] increase the field-star density considerably, thereby decreasing the contrast between cluster and field stars. The result is a larger uncertainty in the background density, $\sigma_{\mathrm{bg}}$, leading to a smaller radius as per Eq. (3) in [B11].

\section{Outlook}

Our study is intended to provide a test-bed for stellar-evolution studies, and will be used to investigate the zero-point calibration of the Galactic PLR for Cepheids. Important limitations to this work, not mentioned here, will be discussed in a forthcoming article.

\section{References}

Baumgardt, H., Dettbarn, C., \& Wielen, R. 2000, A\&AS, 146, 251

Bukowiecki, Ł., et al. 2011, Acta Astronomica, 61, 231 [B11]

Dias, W. S., et al. 2002, A\&A, 389, 871 [DAML]

Fernie, J.D., et al. 1995, IBVS, 4148, 1

Kharchenko, N.V., et al. 2005, A\&A, 438, 1163 [K05]

Robichon, N., et al. 1999, A\&\&A, 345, 471

Samus N. N., et al. 2011, General Catalog of Variable Stars

Sandage, A. \& Tammann, G. A. 2006, ARAESA, 44 (1), 93

Turner, D. G. 2010, APESS, 326 (2), 219

Watson, C. L. 2006, Society for Astronomical Sciences Annual Symposium, 25, 47 